Corrigendum

\title{
Nutritional Supplementation With Physical Activity Improves Muscle Composition in Mobility-Limited Older Adults, The VIVE2 Study: A Randomized, Double-Blind, Placebo-Controlled Trial
}

Davis A. Englund, Dylan R. Kirn, Afsaneh Koochek, Hao Zhu, Thomas G. Travison, Kieran F. Reid, Åsa von Berens, Michael Melin, Tommy Cederholm, Thomas Gustafsson, and Roger A. Fielding

Additional funding information has been added to this article to

clarify the source of funding from the NIH. 